\title{
Dynamic Analysis of an Internal Turning Tool with Elastic Foundation (Winkler Model) ECCOMAS Thematic Conference on Multibody Dynamics 2021
}

\author{
Rouben Rostamian ${ }^{1}$, Wallyson Thomas ${ }^{2}$, Attila Szilagyi ${ }^{3}$ \\ ${ }^{1}$ Department of Mathematics and \\ Statistics \\ ${ }^{2}$ Faculty of Mechanical Engineering and \\ University of Maryland \\ Informatics \\ Baltimore County \\ University of Miskolc \\ Miskolc, 3515, Hungary \\ Baltimore, MD 21250, USA \\ szmwally@uni-miskolc.hu \\ rostamian@umbc.edu \\ ${ }^{3}$ Faculty of Mechanical Engineering and Informatics \\ University of Miskolc \\ Miskolc, 3515, Hungary \\ szilagyi.attila@uni-miskolc.hu
}

\begin{abstract}
This paper focuses on the clamping properties influence on the dynamic properties of clamped boring bars. The boring bar is modeled as a cantilever Euler-Bernoulli beam and a three span configuration in a Winkler foundation, in this way, this theory is applied to derive the transcendental equation for a general case applicable to the system with span beam at an arbitrary location. Eigenvalue plots of the first three modes are presented along with their respective mode shapes. The corresponding natural frequency equations are given and obtained by numerical calculation. The theoretical calculations are validated and discussed. These results confirm that within reason, the theory matches the literature and have relatively approach with the experimental values.
\end{abstract}

Keywords: boring bar, Euler-Bernoulli beam, Winkler foundation, Eigenvalue, natural frequency.

\section{INTRODUCTION}

Consider the transverse displacements $y(x, t)$ at time $t$ of a beam of length $L$ aligned with the interval $0<x<L$ in the Cartesian $x y$ plane. There are several mathematical models of varying complexities that describe the motion $y(x, t)$, the simplest of which is due to Euler and is known as the Euler beam model:

$$
E I \frac{\partial^{4} y}{\partial x^{4}}+\rho A \frac{\partial^{2} y}{\partial t^{2}}=f(x, t)
$$

Here, $A$ is the beam's cross-sectional area, $I$ is the cross-sectional moment of inertia, $\rho$ is the density (i.e., mass per unit volume of the beam), $E$ is the material's Young's modulus, and $f(x, t)$ is the load per unit length applied to the beam.

Each of the derivatives $\partial^{\mathrm{n}} \mathrm{y} / \partial^{\mathrm{n}} \mathrm{x}, \mathrm{n}=1 ; 2 ; 3$, has a physical interpretation and significance:

$\partial \mathrm{y} / \partial \mathrm{x}$ is the beam's slope,

EI $\partial^{2} \mathrm{y} / \partial^{2} \mathrm{x}$ is the bending moment at the cross-section $x$,

EI $\partial^{3} \mathrm{y} / \partial^{3} \mathrm{x}$ is the vertical shear force at the cross-section $x$, 
as illustrated in Fig. 1. Boundary conditions associated with the PDE (1) are determined by translating the physics of the setting through these observations; see Fig. 2.

(a)

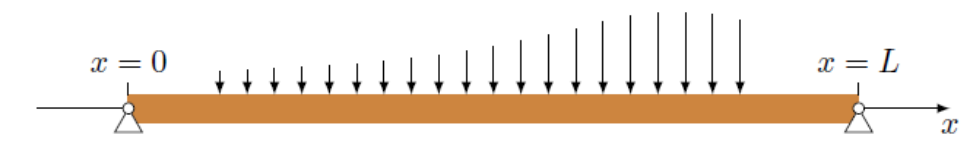

(b)

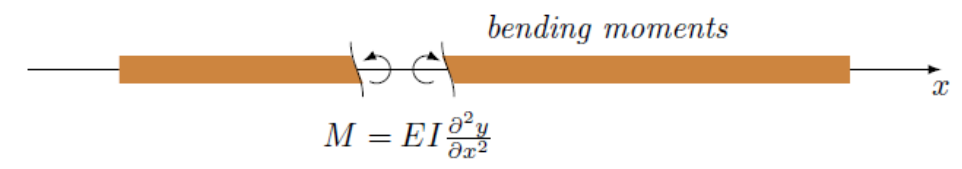

(c)

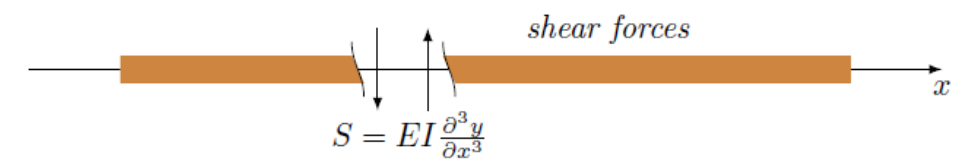

Figure 1. (a) A beam with pinned supports at the ends and a distributed load applied to a part of it. The bending of the beam results in an internal moment $\mathrm{M}$ (Figure (b)) and a transverse shear $\mathrm{S}$ at each cross section (Figure (c)).

(a)

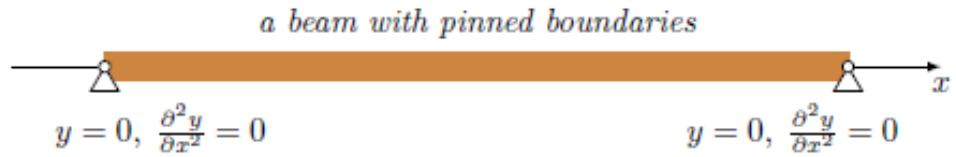

(b)

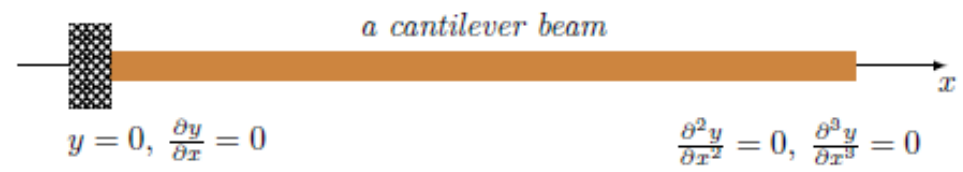

(c)

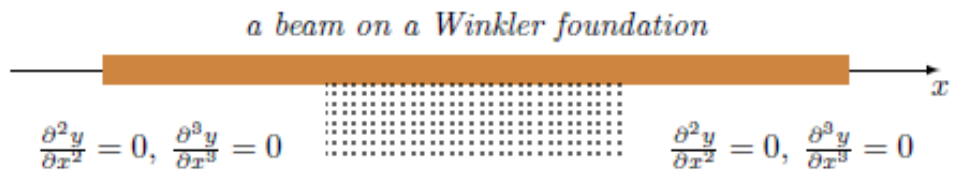

Figure 2. The diagrams show the boundary conditions associated with (a) a beam with pinned supports at the ends; (b) a cantilever beam; (c) a beam partially supported on a Winkler foundation and free otherwise.

\section{WINKLER FOUNDATION}

The earliest formulation of the foundation model was due to Winkler, who assumed the foundation model to consist of closely spaced independent linear springs, as shown in Fig.3. If such a foundation is subjected to a partially distributed surface loading, $q$, the springs will not be affected beyond the loaded region. For such a situation, an actual foundation is observed to have the surface deformation as shown in Fig. 4. Hence by comparing the behavior of theoretical model and actual foundation, it can be seen that this model essentially suffers from a complete lack of continuity in the supporting medium. The load deflection equation for this case can be written as

$$
q=k w
$$

where $k$ is the spring constant and is often referred to as the foundation modulus, and $w$ is the vertical deflection of the contact surface. It can be observed that Equation 2 is exactly satisfied by an elastic plate floating on the surface of a liquid and carrying some load which causes it to deflect. The pressure distribution under such a plate will be equivalent to the force of buoyancy, $k$ being the specific weight of the liquid. With this analogy in view, the first solution for the bending of plates on a Winkler-type foundation was presented by Hertz (1884). 


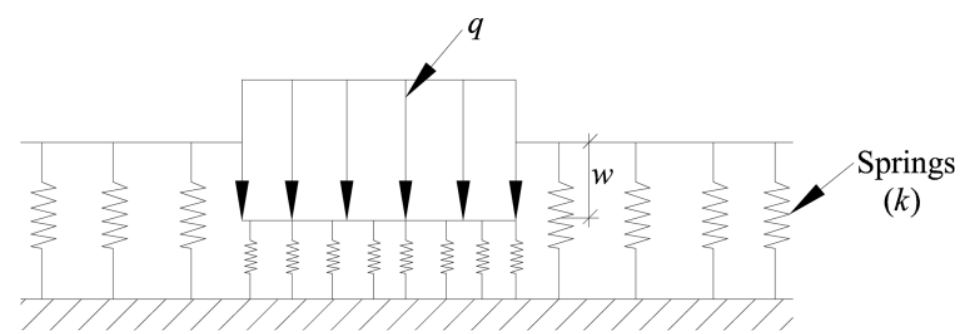

Figure 3. Load on Winkler's foundation
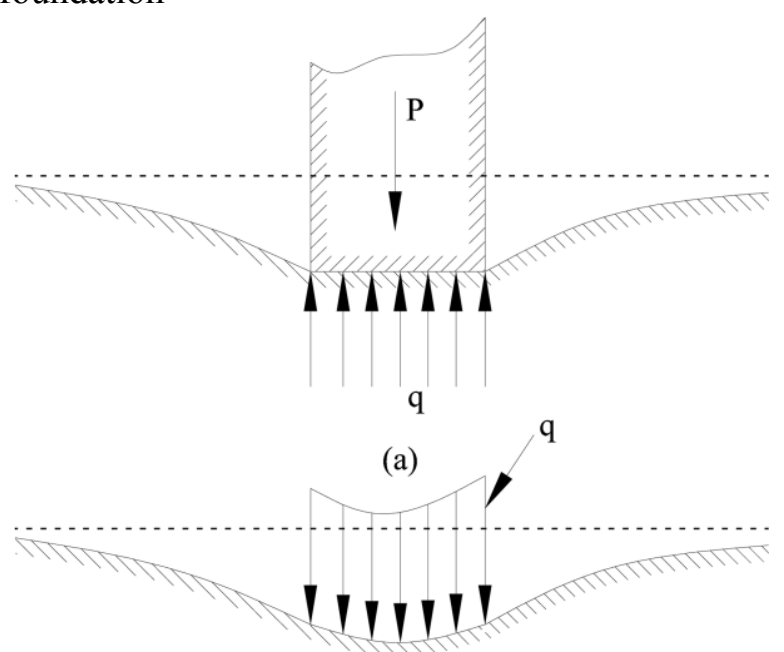

Figure 4. Deformation of actual foundation.

(b)

A Winkler foundation is a distributed springy support, similar to a mattress, that restrains the beam's motion. The Winkler foundation may extend to the entire beam, from end to end, or be limited to a subset of the beam as in Fig. 2(c). We write $K(x)$ for stiffness of the foundation, allowing for the stiffness to vary with $x$. The force exerted by the foundation per unit length of the beam is $-K(x) y(x, t)$, where $y(x, t)$ is the beam's transverse displacement. This leads to the modified form of Euler's equation.

$$
E I \frac{\partial^{4} y}{\partial x^{4}}+\rho A \frac{\partial^{2} y}{\partial t^{2}}=f(x, t)-K(x) y
$$

In the study of a beam's natural frequencies of vibration which is our concern in these notes, the applied load is immaterial. Therefore we let $f(x, t)=0$ from this point onward and focus on the equation:

$$
E I \frac{\partial^{4} y}{\partial x^{4}}+\rho A \frac{\partial^{2} y}{\partial t^{2}}+K(x) y=0
$$

\section{NATURAL FREQUENCY OF VIBRATIONS}

We wish to investigate the natural frequencies of vibrations of an Euler beam of length $\mathrm{L}$ supported on a Winkler foundation. We reduce the equation of motion to a nondimensional form by introducing $\xi=x / L$ as the dimensionless length variable, and write $Y(\xi, t)=y(x, t)$. Then the equation of motion (3) changes to

$$
\rho A \frac{\partial^{2} Y}{\partial t^{2}}+\frac{E I}{L^{4}} \frac{\partial^{4} Y}{\partial \xi^{4}}+K(\xi L)=0, \quad 0<\xi<1, \quad t>0 .
$$

We seek harmonic oscillations of the form $Y(\xi, t)=e^{i \Omega t} u(\xi)$, where $i$ is the unit imaginary number and the angular frequency is to be determined. We substitute this form into Equation (4) and simplify to arrive at

$$
u^{\prime \prime \prime \prime}(\xi)-\frac{\rho A L^{4}}{E I} \Omega^{2} u(\xi)+\frac{K(\xi L) L^{4}}{E I} u(\xi)=0, \quad 0<\xi<1 .
$$


This leads to the introduction of the dimensionless frequency $\omega$ and dimensionless stiffness $\kappa$ through

$$
\omega^{2}=\frac{\rho A L^{4}}{E I} \Omega^{2}, \quad \kappa(\xi)=\frac{K(\xi L) L^{4}}{E I},
$$

Where upon Equation (4) takes the form

$$
u^{\prime \prime \prime}(\xi)-\omega^{2} u(\xi)+\kappa(\xi) u(\xi)=0, \quad 0<\xi<1 .
$$

\section{A CANTILEVER BEAM}

As an exercise, and also to motivate the next section's calculations, let us determine the natural frequencies of vibrations of a cantilever beam in the absence of a Winkler foundation, that is, $\kappa(\xi)$ $=0$ in Equation (8). Thus, referring to Fig. 2(b), we are faced with the boundary value problem

$$
\begin{gathered}
u^{\prime \prime \prime}(\xi)-\omega^{2} u(\xi)=0, \quad 0<\xi<1, \\
u(0)=0, \quad u^{\prime}(0)=0, \quad u^{\prime \prime}(1)=0, \quad u^{\prime \prime \prime}(1)=0 .
\end{gathered}
$$

The characteristic equation of the differential Equation (9) is $r^{4}-\omega^{2}=0$. Therefore $r^{2}= \pm \omega$ (we assume $\omega>0$ without a loss of generality) and conclude that the characteristic roots are $r= \pm \omega^{1 / 2}$ and $r= \pm i \omega^{1 / 2}$, or, letting $\lambda=\omega^{1 / 2}$ to simplify the notation, $r= \pm \lambda$, and $r= \pm i \lambda$. We conclude that the general solution of the differential equation is

$$
u(\xi)=c_{1} \cos \lambda \xi+c_{2} \sin \lambda \xi+c_{3} \cosh \lambda \xi+c_{4} \sinh \lambda \xi
$$

Applying the boundary conditions leads to the equations

$$
\begin{gathered}
c_{1}+c_{3}=0 \\
c_{2}+c_{4}=0 \\
-c_{1} \cos \lambda-c_{2} \sin \lambda+c_{3} \cosh \lambda+c_{4} \sinh \lambda=0 \\
c_{1} \sin \lambda-c_{2} \cos \lambda+c_{3} \sinh \lambda+c_{4} \cosh \lambda=0
\end{gathered}
$$

which we express in the matrix form as

$$
\left[\begin{array}{cccc}
1 & 0 & 1 & 0 \\
0 & 1 & 0 & 1 \\
-\cos \lambda & -\sin \lambda & \cosh \lambda & \sinh \lambda \\
\sin \lambda & -\cos \lambda & \sinh \lambda & \cosh \lambda
\end{array}\right]\left[\begin{array}{l}
c_{1} \\
c_{2} \\
c_{3} \\
c_{4}
\end{array}\right]=\left[\begin{array}{l}
0 \\
0 \\
0 \\
0
\end{array}\right]
$$

Let us write $M(\lambda)$ for the $4 \times 4$ coefficient matrix in (13). This system of four linear homogeneous equation in the four unknowns $c_{1}, c_{2}, c_{3}, c_{4}$ will have a nontrivial

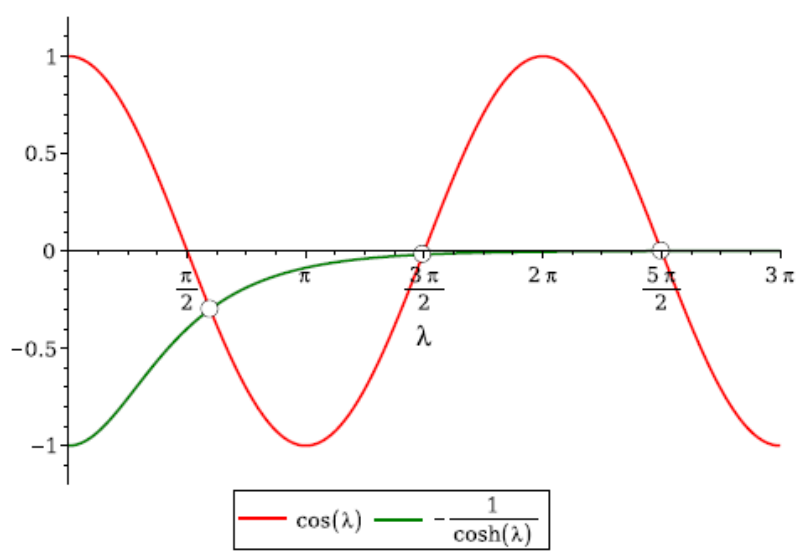

Figure 5. The circles mark the first three roots of the Equation (14). Except for the first, the roots 
essentially agree with those of the cosine function.

solution provided that $\operatorname{det} M(\lambda)=0$. Expanding and simplifying the determinant, leads to the equation

$$
\cos (\lambda)=-\frac{1}{\cosh (\lambda)}
$$

whose roots are depicted graphically in Fig. 5. The first three roots are

$$
\lambda_{1} \approx 1.875104068 \quad \lambda_{2} \approx 4.694091132 \quad \lambda_{3} \approx 7.854757438
$$

Corresponding to each root $\lambda_{\mathrm{i}}, \mathrm{i}=1,2, \ldots$, the system (13) has a nontrivial solution lying within the one-dimensional null space of $M\left(\lambda_{i}\right)$. A numerically robust way of calculating the basis vector of that one-dimensional null space is through the singular value decomposition of $M\left(\lambda_{i}\right)$, that is,

$$
M\left(\lambda_{i}\right)=U S V^{T}
$$

where $U$ and $V$ are orthogonal matrices, and $S$ is the diagonal matrix of $M\left(\lambda_{i}\right)$ 's singular values. See, for instance $[3,4,5,6]$. It turns out, see, e.g., Theorem 5.2 in [5], that the rightmost column of the matrix $V$ is the basis of the null space. Thus, we calculate the null spaces of $M(\lambda)$ corresponding to the first three roots $\lambda_{i}$ and obtain

$$
\left[\begin{array}{l}
c_{1} \\
c_{2} \\
c_{3} \\
c_{4}
\end{array}\right]_{1}\left[\begin{array}{c}
-0.5000 \\
0.3670 \\
0.5000 \\
-0.3670
\end{array}\right] \quad\left[\begin{array}{l}
c_{1} \\
c_{2} \\
c_{3} \\
c_{4}
\end{array}\right]_{2}=\left[\begin{array}{c}
0.5000 \\
-0.5092 \\
-0.5000 \\
0.5092
\end{array}\right] \quad\left[\begin{array}{l}
c_{1} \\
c_{2} \\
c_{3} \\
c_{4}
\end{array}\right]_{3}=\left[\begin{array}{c}
-0.5000 \\
0.4996 \\
0.5000 \\
-0.4996
\end{array}\right]
$$

and therefore, according to (11), the mode shapes are

$$
\begin{aligned}
& u_{1}(\xi)=-0.5 \cos \lambda_{i} \xi+0.3670 \sin \lambda_{i} \xi+0.5 \cosh \lambda_{i} \xi-0.3670 \sinh \lambda_{i} \xi, \quad\left(\lambda_{1}=1.8751\right) \\
& u_{1}(\xi)=-0.5 \cos \lambda_{i} \xi-0.5092 \sin \lambda_{i} \xi-0.5 \cosh \lambda_{i} \xi+0.5092 \sinh \lambda_{i} \xi, \quad\left(\lambda_{2}=4.6941\right) \\
& u_{1}(\xi)=-0.5 \cos \lambda_{i} \xi+0.4996 \sin \lambda_{i} \xi+0.5 \cosh \lambda_{i} \xi-0.4996 \sinh \lambda_{i} \xi, \quad\left(\lambda_{3}=7.8548\right)
\end{aligned}
$$

We have normalized the modes so that $u_{i}(1)=1$ for each mode. That $\mathrm{c}_{1}$ and $\mathrm{c}_{3}$ are $\pm 1 / 2$ is not a coincidence. It can be shown that $c_{1}$ and $c_{3}$ are $\pm 1 / 2$ for all normalized modes for all frequencies. The mode shapes are shown in Fig. 6.

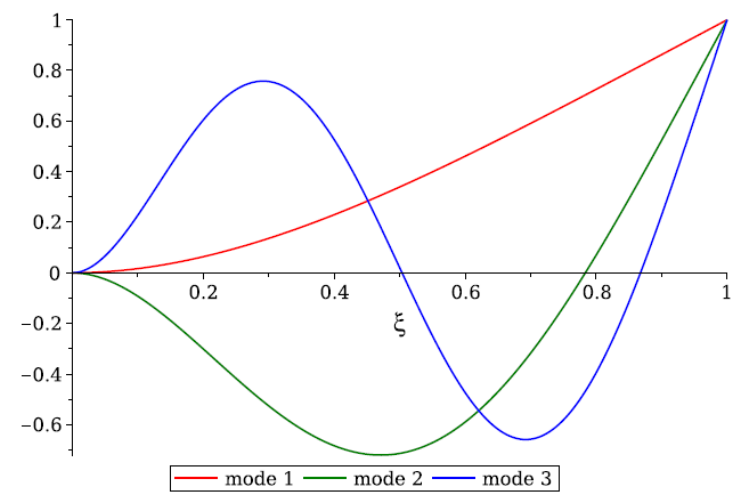

Figure 6. The first three mode shapes of the natural vibrations of a cantilever beam.

\section{A NUMERICAL EXAMPLE - CANTILEVER BEAM}

For this illustration, we consider a cantilever beam with parameter values taken from [1] which is somewhat extended pre-print manuscript corresponding to the published article [2]. (Some of the parameter values have been omitted in the latter.) Specifically, from page 13 of [1] we have

$$
\begin{array}{ll}
A=1.1933 \times 10^{-3} \mathrm{~m}^{2}, & I=1.138 \times 10^{-7} \mathrm{~m}^{4}, \\
\rho=7850 \mathrm{~kg} / \mathrm{m}^{3} & E=205 \times 10^{9} \mathrm{~N} / \mathrm{m}^{2}
\end{array}
$$


and from Section 3.2.1 of [1] we have $L=0.200 \mathrm{~m}$

Let us calculate beam's three lowest natural frequencies. From Equation (14) and referring to Fig. 5 , the three smallest roots are

$$
\lambda_{1} \approx 1.875104068 \quad \lambda_{2} \approx 4.694091132 \quad \lambda_{3} \approx 7.854757438
$$

and therefore

$$
\omega_{1}=\lambda_{1}^{2}=3.516015266 \quad \omega_{2}=\lambda_{2}^{2}=22.03449156 \quad \omega_{3}=\lambda_{3}^{2}=61.69721441
$$

From (5) we have

$$
\Omega=\sqrt{\frac{E I}{\rho A L^{4}}} \omega
$$

Plugging in the data into the above, we conclude that circular frequencies are

$$
\Omega_{1}=4386.611508 \mathrm{~s}^{-1} \quad \Omega_{2}=27490.42508 \mathrm{~s}^{-1} \quad \Omega_{1}=76973.98625 \mathrm{~s}^{-1}
$$

Then we calculate the (ordinary) frequency (oscillations per second) from $f=\Omega /(2 \pi)$ :

$$
f_{1}=698.1509048 \mathrm{~Hz} \quad f_{2}=4375.237039 \mathrm{~Hz} \quad f_{2}=12250.79040 \mathrm{~Hz}
$$

which agree with the data given in Table 3.11 of [1] (and Table 5 of [2]), as expected.

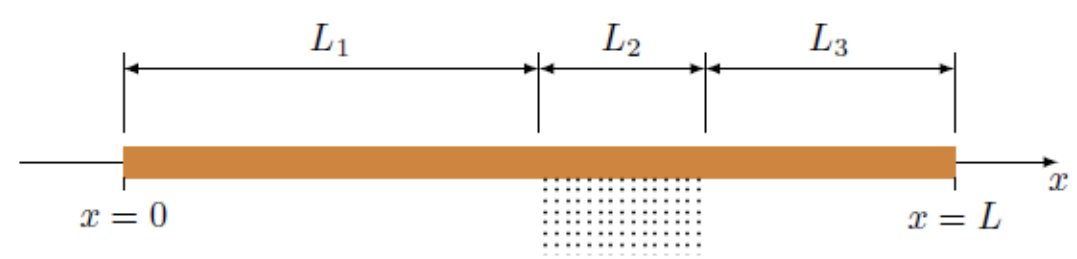

Figure 7. A beam of length $L$ partially supported on a Winkler foundation. The resulting three spans are of lengths $L_{1}, L_{2}$, and $L_{3}$, from left to right. We let $\alpha=L 1=L, \beta=(L 1+L 2)=L$.

\section{A NUMERICAL EXAMPLE - 3 SPAN BEAM}

Fig. 7 depicts a beam of length L supported on a Winkler foundation for a part of its length. We write $L_{1}, L_{2}$, and $L_{3}$ for the lengths of the beam's three segments. As in the previous sections, we introduce the nondimensional length variable $\xi=x / L$, and write $\alpha=L_{1} / L$ and $\beta=(L 1+L 2) / L$ for the (nondimensional) coordinates of the interfaces of the three segments.

We assume that foundation's nondimensional stiffness (see (7)) is a constant $k$ on the supported part, that is, the coefficient $\kappa(\xi)$ of Equation (8) is of the form

$$
\kappa(\xi)=\left\{\begin{array}{lll}
k & \text { if } \quad \alpha<\xi<\beta \\
0 & \text { otherwise }
\end{array}\right.
$$

Consequently, we express the deflection, $u(\xi)$, as a piecewise defined function

$$
u(\xi)=\left\{\begin{array}{lll}
u_{1}(\xi) & \text { if } & 0<\xi<\alpha \\
u_{2}(\xi) & \text { if } & \alpha<\xi<\beta \\
u_{3}(\xi) & \text { if } & \beta<\xi<1
\end{array}\right.
$$

The functions $u_{1}, u_{2}$, and $u_{3}$ are solutions of the equations

$$
\left\{\begin{array}{lll}
u_{1}^{\prime \prime \prime}(\xi)-\omega^{2} u_{1}(\xi)=0 & \text { if } & 0<\xi<\alpha \\
u_{2}^{\prime \prime \prime}(\xi)-\left(\omega^{2}-k\right) u_{2}(\xi)=0 & \text { if } & \alpha<\xi<\beta \\
u_{3}^{\prime \prime \prime}(\xi)-\omega^{2} u_{3}(\xi)=0 & \text { if } & \beta<\xi<1
\end{array}\right.
$$

The general solution of each of these fourth order equations comes with four undefined coefficients. Therefore, the solution of the overall system involves 12 unknown coefficients. The 
physics of the problem supplies 12 boundary and continuity conditions which may be applied to determine the 12 unknowns. Those conditions are

$$
\begin{gathered}
u_{1}^{\prime \prime}(0)=0, \quad u_{1}^{\prime \prime \prime}(0)=0, \\
u_{1}(\alpha)=u_{2}(\alpha), \quad u_{1}^{\prime}(\alpha)=u_{2}^{\prime}(\alpha), \quad u_{1}^{\prime \prime}(\alpha)=u_{2}^{\prime \prime}(\alpha), \quad u_{1}^{\prime \prime \prime}(\alpha)=u_{2}^{\prime \prime \prime}(\alpha), \\
u_{1}(\beta)=u_{2}(\beta), \quad u_{1}^{\prime}(\beta)=u_{2}^{\prime}(\beta), \quad u_{1}^{\prime \prime}(\beta)=u_{2}^{\prime \prime}(\beta), \quad u_{1}^{\prime \prime \prime}(\beta)=u_{2}^{\prime \prime \prime}(\beta), \\
u_{1}^{\prime \prime}(1)=0, \quad u_{1}^{\prime \prime \prime}(1)=0,
\end{gathered}
$$

The boundary conditions (28) set the moment and shear at the endpoint $\xi=0$ to zero, implying that end moves freely. The boundary conditions (31) do the same for the endpoint $\xi=1$. The boundary conditions (30) indicate that the displacement, slope, moment, and shear are continuous at $\xi=\alpha$, and thus, the interface at $\xi=\alpha$ moves free of constraints. The boundary conditions (30) assert the same thing about the interface at $\xi=\beta$.

Equations (27) $)_{1}$ and $(27)_{3}$ are the same as (9), and therefore their solutions are as given in (11), that is

$$
\begin{aligned}
& u_{1}(\xi)=\mathrm{A}_{1} \cos (\sqrt{\omega} \xi)+\mathrm{A}_{2} \sin (\sqrt{\omega} \xi)+\mathrm{A}_{3} \cosh (\sqrt{\omega} \xi)+\mathrm{A}_{4} \sinh (\sqrt{\omega} \xi) \\
& u_{3}(\xi)=\mathrm{C}_{1} \cos (\sqrt{\omega} \xi)+\mathrm{C}_{2} \sin (\sqrt{\omega} \xi)+\mathrm{C}_{3} \cosh (\sqrt{\omega} \xi)+\mathrm{C}_{4} \sinh (\sqrt{\omega} \xi)
\end{aligned}
$$

Solving $(27)_{2}$ requires more e ort since the expression for the general solution depends on the sign of $\omega^{2}-\mathrm{k}$. For that reason, the analysis branches out into three cases.

Case 1: $\omega^{2}>k$. In this case, equation $(27)_{2}$ is qualitatively the same as the two other equations in (27). It is a matter of replacing $\omega^{2}$ by $\omega^{2}-\mathrm{k}$. Therefore, we obtain

$$
\begin{aligned}
& u_{2}(\xi)=\mathrm{B}_{1} \cos \left(\sqrt[4]{\omega^{2}-k} \xi\right)+\mathrm{B}_{2} \sin \left(\sqrt[4]{\omega^{2}-k} \xi\right)+\mathrm{B}_{3} \cosh \left(\sqrt[4]{\omega^{2}-k} \xi\right)+\mathrm{B}_{4} \sinh (\sqrt{\omega} \xi), \\
& \text { (assuming } \left.\omega^{2}>k\right)
\end{aligned}
$$

We apply the 12 boundary conditions (28) to (31) to the solution fragments (32), (33), and (34) and obtain a homogeneous linear system of 12 equations in the 12 unknowns $A_{1}, A_{2}, A_{3}, A_{4}, B_{1}$, $\mathrm{B}_{2}, \mathrm{~B}_{3}, \mathrm{~B}_{4}, \mathrm{C}_{1}, \mathrm{C}_{2}, \mathrm{C}_{3}, \mathrm{C}_{4}$. The system will have a nontrivial solution provided that the determinant of its $12 \times 12$ coefficient matrix is zero. The symbolic calculation of that determinant is quite a demanding task. We computed that in Maple but we do not show the result here since it will fill several printed pages. What needs to be noted here is that the determinant involves the nondimensionalized frequency $\omega$ and stiffness $\mathrm{k}$. By setting the determinant to zero, we may solve for the natural frequencies $\omega$ for any given $\mathrm{k}$.

Having calculated the natural frequencies, the modal shapes are determined as in the case of the cantilever beam in Section 5 by applying the singular value decomposition to the system's $12 \mathrm{x}$ 12 coefficient matrix.

Case 2: $\omega^{2}=k$. In this case, equation $(27)_{2}$ reduces to $u^{\prime \prime \prime}(\xi)=0$ which has a cubic polynomial as its general solution:

$$
u_{2}(\xi)=B_{1}+B_{2} \xi+B_{3} \xi^{3}+B_{4} \xi^{4}
$$

We apply the boundary and interface conditions (13) and obtain a homogeneous linear system of 12 equations in 12 unknowns $\mathrm{A}_{1}, \mathrm{~A}_{2}, \mathrm{~A}_{3}, \mathrm{~A}_{4}, \mathrm{~B}_{1}, \mathrm{~B}_{2}, \mathrm{~B}_{3}, \mathrm{~B}_{4}, \mathrm{C}_{1}, \mathrm{C}_{2}, \mathrm{C}_{3}, \mathrm{C}_{4}$. We set the determinant of the coefficient matrix to zero in order to obtain nontrivial solutions. The determinant involves the foundation stiffness $\mathrm{k}$. Therefore, Case 2 occurs only for special choices of the foundation stiffness. The special values of $\mathrm{k}$ and the corresponding mode shapes may be obtained by a calculation similar to that of Case 1 .

Case 3: $\omega^{2}<k$. Recall that equation $(27)_{2}$ reads

$$
u_{2}^{\prime \prime \prime}(\xi)-\left(\omega^{2}-k\right) u_{2}(\xi)=0
$$


Since $\omega^{2}<\mathrm{k}$, we rewrite it as

$$
u_{2}^{\prime \prime \prime}(\xi)+\left(k-\omega^{2}\right) u_{2}(\xi)=0
$$

The sign change of the parenthesized coefficient has a profound effect on the form of the equation's general solution. Let us write $\lambda^{4}=k-\omega^{2}$. The equation reads $u_{2}{ }^{\prime \prime \prime}{ }^{\prime}+\lambda^{4} u_{2}=0$. The characteristic equation is $r^{4}+\lambda^{4}=0$, which has roots $\lambda=\left[1 /(2)^{1 / 2}\right]( \pm 1 \pm \mathrm{i})$. It follows that the differential equation's general solution is

$$
\begin{aligned}
& u_{2}(\xi)=B_{1} \cosh \frac{\lambda}{\sqrt{2}} \xi \cos \frac{\lambda}{\sqrt{2}} \xi+B_{2} \sinh \frac{\lambda}{\sqrt{2}} \xi \cos \frac{\lambda}{\sqrt{2}} \xi+ \\
& B_{3} \cosh \frac{\lambda}{\sqrt{2}} \xi \sin \frac{\lambda}{\sqrt{2}} \xi+B_{4} \sinh \frac{\lambda}{\sqrt{2}} \xi \sin \frac{\lambda}{\sqrt{2}} \xi
\end{aligned}
$$

where $\lambda=\left(k-\omega^{2}\right)^{1 / 4}$. The calculation of the eigenfrequencies $\omega$ proceeds as in in previous cases.

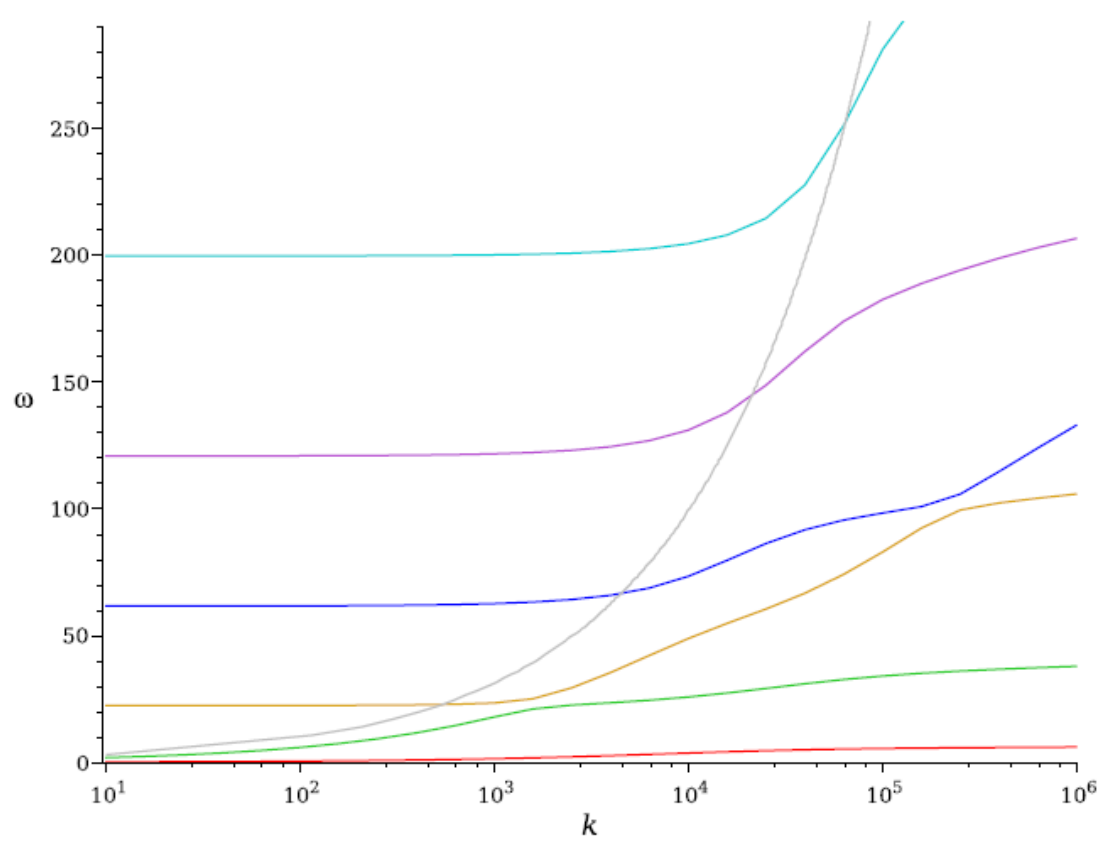

Figure 8. The frequencies $\omega$ plotted against the foundation stiffness $k$ for the Winkler-supported beam of Figure 7. The gray curve is the graph of $\omega^{2}=\mathrm{k}$ that separates the three cases considered in Section 6.

\section{NUMERICAL CALCULATIONS OF A THREE-SPAN WINKLER-SUPPORTED BEAM}

We apply an appropriately modified version of Section 5's numerical data to the Winklersupported beam. Following [1] and [2], we take

$$
\begin{aligned}
& L_{1}=0.215 \mathrm{~m}, \quad L_{2}=0.050 \mathrm{~m}, \quad L_{3}=0.035 \mathrm{~m} \\
& A=1.1933 \times 10^{-3} \mathrm{~m}^{2}, \quad I=1.138 \times 10^{-7} \mathrm{~m}^{4}, \\
& \rho=7850 \mathrm{~kg} / \mathrm{m}^{3}, \quad E=205 \times 10^{9} \mathrm{~N} / \mathrm{m}^{2},
\end{aligned}
$$

whence $L=L 1+L 2+L 3=0.300 \mathrm{~m}$, and $\alpha=215 / 300, \beta=265 / 300$. We allow for a wide range of the (non-dimensionalized) foundation stiffness by letting $k$ range from $10^{1}$ to $10^{6}$. For $k$ in that range we calculate the beam's natural frequencies $\omega$. Fig. 8 shows the results for the first six vibration modes.

The calculation that produces that diagram is quite nontrivial due to the fact that the determinant of the coefficient matrix is not only an exceedingly large transcendental expression, but that is oscillates wildly as a function of $\mathrm{k}$, taking values in the range $10^{-80}$ to $10^{-80}$. As a result, calculating 
the roots of the determinant is not feasible in the usual hardware floating point arithmetic of a typical computer which handles roughly 16 to 17 significant digits. Attempting to calculate the roots in hardware floating point either produces no result (no convergence) or produces junk. We performed the calculations in Maple's software floating point arithmetic with 70 significant digits and verified that a further increase of the number of significant digits does not affect the results in an appreciable way.

The specific beam analyzed in $[1,2]$ is just like the beam considered above, but instead of a Winkler foundation, the beam is held in place with three pairs of torqued screws. The screws are elastic and therefore allow for some transverse displacement of the beam, somewhat similar to our Winkler foundation. The spring constants $\mathrm{kT}$ of the screws are given in Table 2.6 of [1] (Table 3 of [2]) as $\mathrm{kT}=4.881 \times 10^{9} \mathrm{~N} / \mathrm{m}$ and $\mathrm{kT}=7.732 \times 10^{9} \mathrm{~N} / \mathrm{m}$, depending on the type of the screw. For the present calculations, we take an intermediate value of $\mathrm{kT}=6 \times 10^{9} \mathrm{~N} / \mathrm{m}$. There are three pairs of such retaining screws acting along the length $L_{2}=0.050 \mathrm{~m}$. If we distribute the force exerted by the screws uniformly along the length $L_{2}$, we obtain the Winkler foundation's stiffness as

$$
K=\frac{3 \times\left(6 \times 10^{9}\right)}{0.05}=3.6 \times 10^{11} \mathrm{~N} / \mathrm{m}^{2}=3.6 \times 10^{11} \mathrm{~Pa}
$$

and then, according to (5), the dimensionless stiffness is

$$
\kappa=\frac{K L^{4}}{E I}=\frac{\left(3.6 \times 10^{11}\right) \times 0.3^{4}}{\left(205 \times 10^{9}\right) \times\left(1.138 \times 10^{-7}\right)}=124994.64 \approx 1.25 \times 10^{5}
$$

The non-dimensional natural frequencies $\omega$ of such a beam may be read off of the graphs in Fig. 8 , or for greater accuracy, calculated directly. We find the first three frequencies to be

$$
\omega_{1}=5.569355, \omega_{2}=34.655339, \quad \omega_{3}=87.598947,
$$

and then calculate the actual circular frequencies from (11) as:

$$
\Omega_{1}=3088.167304 \mathrm{~s}^{-1}, \quad \Omega_{2}=19216.13583 \mathrm{~s}^{-1}, \quad \Omega_{3}=48572.98441 \mathrm{~s}^{-1},
$$

Finally, we calculate the (ordinary) frequency (oscillations per second) from $f=\Omega / 2 \pi$ :

$$
f_{1}=491.497, \quad f_{2}=3058.343, \quad f_{3}=7730.630,
$$

These frequencies are somewhat lower than those presented in Table 3.13 of [1] (Table 5 of [2]), which is expected, since a Winkler foundation allows greater flexibility compared to clamping screws.

Fig. 7 depicts the mode shapes corresponding to these frequencies.

\section{CONCLUSION}

It was possible to model a fixation of the tooling system by applying Euler Bernoulli beam model with Winkler Foundation. Furthermore, costly and time-consuming experiments could be replaced by even analytical modelling with manual calculations, which enables us to judge the influence of the geometrical dimensions, the material properties, the possible overhangs and the fixation dimensions of the tool-holder system.

\section{ACKNOWLEDGMENTS}

This research was supported by the European Union and the Hungarian State, co-financed by the European Regional Development Fund in the framework of the GINOP-2.3.4-15-2016-00004 project, aimed to promote the cooperation between the higher education and the industry.

\section{REFERENCES}

[1] Henrik_Akesson, Tatiana Smirnova, and Lars H_akansson. Analysis of dynamic properties of boring bars concerning different clamping conditions. Preprint manuscript, 2007. 
[2] Henrik Akesson, Tatiana Smirnova, and Lars Hakansson. Analysis of dynamic properties of boring bars concerning di erent clamping conditions. Mechanical Systems and Signal Process-ing, 23:2629\{2647, 2009.

[3] Kendall E. Atkinson. An Introduction to Numerical Analysis. Wiley, New York, 2 edition, 1989.

[4] David Kincaid and Ward Cheney. Numerical Analysis: Mathematics of Scienti_c Computing. American Mathematical Society, Providence, RI, 3 edition, 2002.

[5] Carl Meyer. Matrix analysis and applied linear algebra. Society for Industrial and Applied Mathematics (SIAM), Philadelphia, PA, 2000.

[6] Lloyd N. Trefethen and David Bau, III. Numerical linear algebra. Society for Industrial and Applied Mathematics (SIAM), Philadelphia, 1997. 